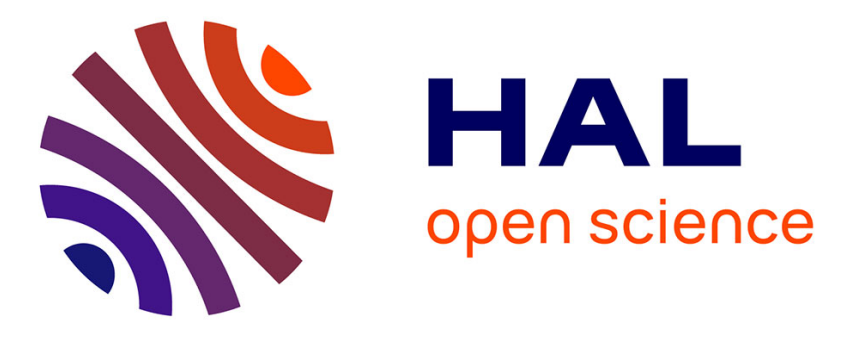

\title{
Pristane-induced arthritis in dark Agouti rat is a relevant model for mimicking vascular dysfunction and lipid paradox in rheumatoid arthritis
}

Mickaël Chouk, Romain Bordy, Johnny Moretto, Daniel Wendling, Perle Totoson, Céline Demougeot

\section{To cite this version:}

Mickaël Chouk, Romain Bordy, Johnny Moretto, Daniel Wendling, Perle Totoson, et al.. Pristane-induced arthritis in dark Agouti rat is a relevant model for mimicking vascular dysfunction and lipid paradox in rheumatoid arthritis. Joint Bone Spine, 2019, 86, pp.483 - 490. 10.1016/j.jbspin.2018.12.001 . hal-03486433

\section{HAL Id: hal-03486433 \\ https://hal.science/hal-03486433}

Submitted on 20 Dec 2021

HAL is a multi-disciplinary open access archive for the deposit and dissemination of scientific research documents, whether they are published or not. The documents may come from teaching and research institutions in France or abroad, or from public or private research centers.
L'archive ouverte pluridisciplinaire HAL, est destinée au dépôt et à la diffusion de documents scientifiques de niveau recherche, publiés ou non, émanant des établissements d'enseignement et de recherche français ou étrangers, des laboratoires publics ou privés.

\section{(ㄷ)(1) $\$$}

Distributed under a Creative Commons Attribution - NonCommerciall 4.0 International 


\title{
Pristane-Induced Arthritis in Dark Agouti rat is a relevant model for mimicking vascular dysfunction and lipid paradox in rheumatoid arthritis
}

\author{
Mickaël Chouk $^{\mathrm{a}, \mathrm{b}}$, Romain Bordy ${ }^{\mathrm{a}}$, Johnny Moretto ${ }^{\mathrm{a}}$, \\ Daniel Wendling ${ }^{\mathrm{b}, \mathrm{c}, \#}$, Perle Totoson ${ }^{\mathrm{a}}$, Céline Demougeot ${ }^{\mathrm{a}}$
}

${ }^{a}$ PEPITE EA4267, FHU INCREASE, Univ. Bourgogne Franche-Comté, 25000 Besançon, France

${ }^{\mathrm{b}}$ Service de Rhumatologie, CHRU Besançon, 25000 Besançon, France

${ }^{c}$ EA 4266, Univ. Bourgogne Franche-Comté, 25000 Besançon, France

\section{\# Corresponding author:}

Daniel Wendling

CHRU Besançon, Service de Rhumatologie, 3 boulevard Alexandre Fleming, 25000 Besançon, France

Tel: (33) 381668241

E-mail address: dwendling@ @hu-besancon.fr 


\section{Abstract}

Objectives. To understand the pathophysiology of cardiovascular (CV) dysfunction in rheumatoid arthritis (RA) is crucial, but limited by the paucity of animal models able to mimic CV impairments. We wanted to determine if the rat model of Pristane-Induced Arthritis (PIA) reproduced cardiometabolic impairments of RA.

Methods. Dark Agouti rats received an injection of pristane or saline (controls) at day 0. Reactivity to vasoconstrictors and vasodilators was studied in aortic rings and mesenteric arteries at day 28 (acute) and day 120 post-induction (chronic phase). Circulating markers of inflammation, lipid and glucose levels, arthritis and radiographic scores were assessed.

Results. In aortic rings, PIA induced a reduced vasoconstriction to phenylephrine and serotonin in both phases of the model. The relaxant effect of acetylcholine was decreased in PIA in acute $(p<0.05)$ but not in chronic phase. In mesenteric arteries, only the acetylcholineinduced vasorelaxation was impaired in PIA rats in the chronic phase $(p<0.001)$. Serum interleukin-6 levels were higher, total cholesterol and triglycerides levels were lower in PIA in both phases $(p<0.001)$ whereas myeloperoxidase activity and blood glucose were unchanged. Adiponectine levels were lower in PIA in acute $(p<0.001)$ but not in chronic phase. Endothelial function correlated with interleukin-6, total cholesterol levels and arthritis score in aorta but not in mesenteric arteries.

Conclusions. As new information, PIA induces endothelial dysfunction in micro/macrovascular beds and low lipid levels, like in RA. This model of chronic arthritis might be useful to study $\mathrm{CV}$ pathophysiology and to screen new therapeutic options for reducing $\mathrm{CV}$ risk in $\mathrm{RA}$.

Keywords: chronic arthritis - pristane - vascular dysfunction - rat 


\section{Introduction}

Chronic inflammatory rheumatic diseases, including rheumatoid arthritis (RA), are associated with increased cardiovascular (CV) morbidity and mortality $(1,2)$. In RA, the risk of CV mortality is increased by $50 \%$ compared to general population (3), and persists even after adjusting for $\mathrm{CV}$ risk factors (4). The relative increase in risk of myocardial infarction and stroke in RA is $68 \%$ and $41 \%$, respectively (3). The mechanisms linking RA to high CV mortality are still unknown but immune dysregulation, inflammation, metabolic disturbances, drugs and probably other yet unidentified factors are likely to affect CV health through the development of endothelial dysfunction (ED). ED is a functional and reversible alteration of endothelial cells that leads to a shift in the properties of the endothelium towards reduced vasodilatation, a pro-inflammatory state, and pro-proliferative and prothrombotic properties. In RA, ED can occur in large vessels (such as conduit arteries) where it is an obligatory step for the development of atheroma, and in small vessels of the microvasculature which are involved in end-organ damages (5). Clinical evidence indicated that ED is present in early and established RA (6), and suggested that macrovascular ED is linked to inflammation whereas microvascular ED is not (5). As regards the metabolic impairments, RA is associated with a "lipid paradox" characterized by a paradoxical increased CV risk in patients with decreased lipid levels (7).

Despite remarkable improvements in RA treatment, the CV mortality gap between patients and general population is not closing (8), indicating that a better understanding of the pathophysiology of CV impairments in RA is required. Thus, there is a need of animal models of arthritis able to mimic the main features of the cardiometabolic dysfunction in RA (9). Such models would allow to overcome the unavoidable limitations of clinical including heterogeneity in disease severity and duration in RA cohorts, lack of control groups, high level of polymedication including $\mathrm{CV}$ drugs which can impact upon endothelial function. 
Data from the widely-used model of Collagen-Induced Arthritis (CIA) in mice revealed controversial results as regards the presence of ED $(10,11)$. By contrast, the Adjuvant-Induced Arthritis (AIA) model in rat appeared relevant for mimicking ED in macro- and microvasculature in RA (12). However, the AIA model do not exhibit dyslipidemia (13), and only develop a "monophasic" arthritis, making this model not totally appropriated for longterm studies on CV impairments.

The aim of this study was to determine if the Pristane-Induced Arthritis (PIA) model in rat is useful for mimicking vascular dysfunction and lipid impairments in RA. This model in rat is a model of polyarthritis characterized by a chronic disease course (14), the presence of rheumatoid factor (RF) $(15,16)$, and a good response to methotrexate, anti-TNF $\alpha$ and glucocorticoids $(14,17,18)$. Vascular function (endothelial function and response to vasoconstrictive agents) was studied in isolated aortic rings (macrovascular bed) and in third order mesenteric arteries (microvascular bed) in PIA rats in both acute and chronic phases of the model. Blood markers of metabolic impairments such as cholesterol, triglycerides glucose and adiponectin levels were measured. Circulating inflammation was assessed through the measure of interleukin-6 (IL-6) levels and myeloperoxidase activity. The severity of arthritis was evaluated by clinical and radiological scores.

\section{Methods}

\subsection{Animals}

Eighty Dark Agouti male rats aged of 9-10 weeks were purchased from Envigo (Gannat, France). Animals were kept under a $12 \mathrm{~h}-12 \mathrm{~h}$ light/dark cycle and allowed free access to food and water. The experimental procedures were approved by the local committee for ethics in animal experimentation $\mathrm{n}^{\circ}$ 2015-001-CD-5PR of Franche-Comté University (Besançon, 
France), and complied with the "Animal Research: Reporting In Vivo Experiments" ARRIVE guidelines.

\subsection{Arthritis induction, clinical evaluation, disease course definitions}

After anesthesia with isoflurane and application of alcohol at the base of the tail to separate hairs, $150 \mu$ of pristane (2,6,10,14-Tetramethylpentadecane, Sigma) were infused intradermally on the dorsal surface of the base of the tail (14). Control rats received the same volume of saline. Body weight and arthritis score were daily monitored. Arthritis score was determined by monitoring erythema and edema for all paws: every toe/knuckle received a score up to 1 point and every mid-paw or ankle received a score up to 5 points $(14,17)$. The maximum score for each rat is 60 .

The onset of the acute arthritis phase was defined by the first day with an arthritis score greater than or equal to $1 / 60$ (14). The remission phase corresponded to the period between the end of inflammatory symptoms of acute arthritis phase and the onset of chronic arthritis phase. The onset of the chronic arthritis phase was defined after day 60 by the first day with an arthritis score equal to 5 if the mean arthritis score at the end of the follow-up was greater than or equal to 5 , or by the first day with an arthritis score greater than or equal to 6 during 2 consecutive days minimum if the mean arthritis score at the end of follow-up was not greater than or equal to 5 (14). Forty rats were studied in acute phase (20 PIA / 20 controls) and 40 in chronic phase (20 PIA / 20 controls).

\subsection{Radiological analysis}

Hind paws X-rays were completed with the Block Matching Algorithm High Resolution Digital X Ray (40mV, 10mA) (D3A Medical Systems, France). A score of 0-20 was determined for each paw taking into account soft tissues swelling, osteoporosis, loss of 
cartilage, heterotopic ossification and bone erosions (19). The following scale was used: 0 (normal), 1 (low), 2 (medium), 3 (moderate), 4 (severe) for each parameters. The maximum score for each rat is 40 .

\subsection{Tissue collection}

Twenty-eight (acute phase) or 120 (chronic phase) days after PIA induction, rats were anesthetized with pentobarbital (60 mg/kg, i.p., Ceva Santé Animale, France). Blood was withdrawn from the abdominal aorta to obtain serum and plasma, divided into aliquots and stored at $-80^{\circ} \mathrm{C}$ until analysis. Thoracic aortas and mesenteric arteries were removed and immediately used for vascular reactivity studies.

\subsection{Macrovascular reactivity}

Thoracic aorta was excised, cleaned of connective tissue, and cut into rings of $\sim 2 \mathrm{~mm}$ in length. Rings were suspended in Krebs solution (mM: $\mathrm{NaCl} 118, \mathrm{KCl} 4.65, \mathrm{CaCl}_{2} 2.5$, $\mathrm{KH}_{2} \mathrm{PO}_{4} 1.18, \mathrm{NaHCO}_{3} 24.9, \mathrm{MgSO}_{4} 1.18$, glucose 12 ), maintained at $37^{\circ} \mathrm{C}$ and $\mathrm{pH} 7.4$ and continuously aerated with $95 \% \mathrm{O}_{2} / 5 \% \mathrm{CO}_{2}$, for isometric tension recording in isolated organ baths (emkaBATH4 v2.1, Emka Technologie, data acquisition software IOX v2.8). Rings were gradually stretched from $0.5 \mathrm{~g}$ to $2 \mathrm{~g}$ (base tension) in $60 \mathrm{~min}$. Then, to test viability, rings were contracted by concentrated $\mathrm{KCl}$ Krebs solution $(100 \mathrm{mM})$. To assess the endothelium integrity, rings were contracted with serotonin (5-HT) and then relaxed with acetylcholine (Ach). Endothelium integrity was defined by a relaxation percentage greater than $80 \%$. In some rings, endothelium was mechanically removed. The completeness of endothelial denudation was attested by a relaxation percentage less than $10 \%$. We studied vasoconstriction on endothelium-intact and endothelium-denuded rings with increasing 
concentrations of different pharmacological agents: phenylephrine (Phe, $10^{-11}$ to $10^{-4} \mathrm{M}$ ) and 5-HT $\left(10^{-11}\right.$ to $\left.10^{-4} \mathrm{M}\right)$. Macrovascular endothelial function was measured in endotheliumintact aortic rings by measuring the vasorelaxant response to cumulative concentrations of Ach $\left(10^{-11}\right.$ to $\left.10^{-4} \mathrm{M}\right)$ on pre-contracted rings with equipotent concentrations of 5-HT. To determine the sensitivity to nitric oxide (NO) of vascular smooth muscle cells (VSMC), rings were exposed to increasing concentrations of a NO donor, sodium nitroprusside (SNP, $10^{-11}$ to $\left.10^{-4} \mathrm{M}\right)$

\subsection{Microvascular reactivity}

Third order mesenteric artery was excised, cleaned of connective tissue, and cut into rings of $\sim 2 \mathrm{~mm}$ in length, suspended in Krebs solution, maintained at $37^{\circ} \mathrm{C}$ and $\mathrm{pH} 7.4$, and continuously aerated with $95 \% \mathrm{O}_{2} / 5 \% \mathrm{CO}_{2}$. Rings were stretched between two $25 \mu \mathrm{m}$ tungsten wires for isometric tension recording in isolated organ baths (MultiWire Myograph System 610M and 620M, DMT, data acquisition software Labchart7). Mesenteric rings were gradually stretched to a transmural pressure at $90 \%$ of $100 \mathrm{mmHg}$ (base tension) after $30 \mathrm{~min}$ of rest. Then, to test viability, rings were contracted by concentrated $\mathrm{KCl}$ Krebs solution (100 $\mathrm{mM})$. Endothelium integrity was evaluated by the relaxation of segments contracted with Phe and relaxed with Ach. Vasoconstrictive reactivity to Phe $\left(10^{-8}\right.$ to $\left.10^{-4} \mathrm{M}\right)$ and $5-\mathrm{HT}\left(10^{-8}\right.$ to $10^{-}$ ${ }^{4}$ M) was studied. Endothelial function was evaluated by the vasorelaxant response to cumulative concentrations of Ach $\left(10^{-8}\right.$ to $\left.10^{-4} \mathrm{M}\right)$ on segments pre-contracted with Phe $\left(3 \times 10^{-}\right.$ $\left.{ }^{6} \mathrm{M}\right)$. The response to SNP $\left(10^{-8}\right.$ to $\left.10^{-4} \mathrm{M}\right)$ was evaluated by exposure of preconstricted rings.

\subsection{Blood measurements}


Levels of IL-6 and adiponectin were measured in serum by using ELISA kits (PromoKine/PromoCell, Germany and Assaypro, USA, respectively). The limits of detection provided by the manufacturer were $31.2 \mathrm{pg} / \mathrm{ml}$ and $1.1 \mathrm{ng} / \mathrm{ml}$, respectively. Total cholesterol and triglycerides were measured in serum by using assay kits (abcam, England, detection limits: $20 \mu \mathrm{g} / \mathrm{ml}$ and $40 \mathrm{nmol} / \mathrm{ml}$, respectively) and glucose was measured in blood by using glucometer (FreeStyle Papillon Vision, Abbott, France). Plasma myeloperoxidase (MPO) activity was determined by O-dianisidine $\mathrm{H}_{2} \mathrm{O}_{2}$ method as previously described (20) with some modifications. Briefly, $50 \mu \mathrm{l}$ of plasma were add to $100 \mu \mathrm{l}$ of $50 \mathrm{mM}$ potassium phosphate buffer ( $\mathrm{pH}$ 6.0) containing $0.5 \%$ hexadecyltrimethylammonuim bromide, chilled in an ice bath for $10 \mathrm{~min}$ and then incubated at $25^{\circ} \mathrm{C}$ for $20 \mathrm{~min}$. MPO activity was measured spectrophotometrically $(\lambda: 460 \mathrm{~nm})$ after adduction of $\mathrm{H}_{2} \mathrm{O}_{2}$ and expressed as nmol Odianisidine oxidized. $\mathrm{min}^{-1}$.

\subsection{Statistical analysis}

Values are presented as means \pm SEM. Data were analyzed by using GraphPad Prism (version 5.3) and SigmaStat (version 3.5) software. Contractile responses to Phe and 5-HT were expressed as the percentage of the maximum response to $\mathrm{KCl} 100 \mathrm{mM}$. Vasorelaxant responses to Ach and SNP were expressed as the percentage of relaxation of the contractile response to Phe or 5-HT. Effect-concentration curves to each pharmacological drug were compared by 2-way analysis of variance (ANOVA) for repeated measures. Comparisons between two values were assessed by unpaired Student $t$ test or Mann-Whitney $U$ test when data were not normally distributed. The analysis of the relationship between two parameters was determined by linear regression analysis and Spearman correlation coefficient was calculated between these variables. $\mathrm{P}<0.05$ was considered statistically significant. 


\section{Results}

\subsection{Disease course in PIA}

PIA rats developed an acute arthritis phase at day 13 post-induction. A body weight loss was concomitant to the first arthritis symptoms. Then, arthritis progressed to a maximal score on day 20. The remission phase was observed between day 45 and day 73 with a low arthritis score. The chronic arthritis phase began on day 74 with a high heterogeneity for the day of beginning (between day 48 and day 116) and the severity of arthritis (scores between 7 and 27). No further remission phase was observed up to the end of follow-up (day 120) (Figure 1, Table 1). Interestingly, there was no correlation between the maximum arthritis score in acute phase and the minimum score in remission phase $(\mathrm{r}=-0.105, p=0.715)$, neither between the minimum score in remission phase and the maximum score in chronic phase $(r=0.427$, $p=0.121$, and nor between the maximum score in acute phase and the maximum score in chronic phase $(\mathrm{r}=0.194, p=0.492)$. Finally, the maximum score in acute phase was not different between rats that developed a chronic phase and those who did not $(p=0.073)$.

\subsection{Structural course in PIA}

In both arthritis phases, the radiographic score was higher in PIA compared to controls (Figure 1, Table 1). In acute phase, swelling of soft tissues was predominant and there was also heterotopic ossification in front of tarsus [Appendix A, Figure S1; See the supplementary material associated with this article online]. Tarsus and tibio-talus joints were the most affected 
joints, although there was no high bone or cartilage destruction. Osteoporosis was minimal or non-evident. The radiographic score was positively correlated with the arthritis score $(\mathrm{r}=0.843, p<0.0001)$. In chronic phase, the radiographic score was higher compared to acute phase $(p<0.001)$ and correlated with the arthritis score $(\mathrm{r}=0.899, p<0.0001)$. Damages were destructive and constructive with a predominance at tarsus (Appendix A, Figure S1). Tibiotalus joint may also be affected. Metatarsophalangeal and interphalangeal joints seemed to be spared. There was a calcaneal involvement with a calcaneus of greater volume and bone density. In addition to tarsal heterotopic ossification, extra-articular new bone formation can be observed in main body of Achilles tendon.

\subsection{PIA is associated with increased levels of circulating markers of inflammation and lipid disorders but not with glycemic disorders}

As shown in Table 2, compared to controls, circulating IL-6 levels were higher in PIA whatever the phase. A trend towards higher MPO activity was observed in PIA at both phases, but the difference did not reach significance. Blood glucose was unchanged in PIA whatever the phase. By contrast, triglycerides and total cholesterol levels were decreased in PIA at both phases. Adiponectin levels were lower in PIA in acute phase whereas no more difference was observed in chronic phase.

\subsection{PIA induces a vascular dysfunction}

3.4.1. Reactivity to vasoconstrictors is impaired in macrovascular but not in microvascular bed 
In both phases, the vasoconstrictive effect of Phe was dramatically lower in PIA compared to controls in aortic rings (Figures 2A-B). Likewise, the response to 5-HT was lower in PIA but the difference was less marked than for Phe (Figures 2C-D).

To understand the origin of the altered response to Phe and 5-HT in PIA, the effectconcentrations curves of the two drugs were repeated on endothelium-denuded aortic rings (Appendix A, Figure S2). In both phases, the vasoconstrictive effect of Phe was lower in PIA rats compared to controls, indicating that this abnormal response to Phe originated from an impairment of both VSMC and endothelial cells. Conversely, the vasoconstrictive effect of 5HT was not different between groups in both phases.

Contrary to macrovascular bed, in both phases, the vasoconstrictive effects of Phe and 5-HT on mesenteric arteries were no different in PIA compared to controls (Figures 2E-H).

\subsubsection{Macrovascular endothelial dysfunction is present in acute but not in chronic phase of PIA}

In aortic rings, as a reflection of $\mathrm{ED}$, the Ach-induced vasorelaxation was lower in PIA compared to controls in acute phase (Figure 3A). By contrast, no difference was observed between PIA and controls in chronic phase (Figure 3B). To exclude an altered response of the VSMC to NO produced after Ach stimulation, the effect of SNP was studied. The vasorelaxation induced by SNP was not different between groups in both phases (Figures 3CD).

3.4.3. Microvascular endothelial dysfunction is present in chronic but not in acute phase of PIA 
In mesenteric arteries, the Ach-induced vasorelaxation was not different between PIA and controls in acute phase (Figure 3E), whereas it was significantly lower in PIA compared to controls in chronic phase (Figure 3F). The response to SNP was not different between groups in both phases (Figures 3G-H).

\subsubsection{Correlations between endothelial function, arthritis score, radiographic score, and circulating markers}

A correlation analysis between endothelial function, assessed by the response to Ach (Emax), and other parameters was made in all the PIA rats studied in acute and chronic phases (Appendix A, Figure S3). Arthritis score negatively correlated with macrovascular endothelial function $(\mathrm{r}=-0.557, p<0.001, \mathrm{n}=33)$ but not with microvascular endothelial function $(\mathrm{r}=-$ $0.039, p=0.86, \mathrm{n}=22$ ). Likewise, IL-6 levels negatively correlated with endothelial function in macrovascular $(\mathrm{r}=-0.501, p<0.05, \mathrm{n}=25)$ but not in microvascular bed $(\mathrm{r}=-0.261, p=0.249$, $\mathrm{n}=21$ ). Total cholesterol levels also correlated with endothelial function in macrovascular $(\mathrm{r}=0.417, p<0.05, \mathrm{n}=30)$ but not in microvascular bed $(\mathrm{r}=0.071, p=0.747, \mathrm{n}=22)$. By contrast, no correlation was found between macro- and microvascular endothelial functions and circulating MPO activity, adiponectin and triglycerides levels or radiographic score.

\section{Discussion}

Although CV disease is acknowledged as the leading cause of death in RA patients, only a few animal models exist to mimic the features of CV dysfunction in this disease. This study provides the new information that the PIA model reproduces some important features of RA including the presence of an ED in both macro- and microvascular beds, a difference among 
vascular beds with respect to the course of ED and the link with inflammation, as well as disturbances in lipid levels mimicking the "lipid paradox".

Developed in 1990s (21), the PIA model in rat is a model of polyarthritis $(14,21)$ characterized by a chronic disease course (14), the involvement of CD4+ T cells and also B cells, reflected by the presence of $\mathrm{RF}(15,16)$. This last feature is important for the study of CV dysfunction because RF could be a predictor of CV events in RA (22). In this study, induction of the PIA model leads to three characteristic phases, as previously described $(14,17)$. While the acute phase was reproducible in terms of frequency, day of beginning and severity, there was a heterogeneity for the beginning and the severity of arthritis in chronic phase, consistent with previous results (14). We determined that the heterogeneity in chronic phase was not related to the disease activity in acute nor in remission phases. To our knowledge, this work is the first to carry out a specific radiographic study of hind paws in PIA at different phases. Interestingly, joint damages observed in PIA in acute phase (radiographic score at $20 \%$ of maximum score) were lower than those observed in other arthritis models such as AIA (radiographic score at $60 \%$ of maximum score at day 33) (23), or CIA in mice (radiographic score at $40 \%$ of maximum score at day 27) (24). As observed in other models, bone neoformation is important in PIA, which makes it different from RA which is mainly erosive. However, the correlation between arthritis and radiographic scores at both phases in PIA mimics RA in which persistent joint inflammation leads to its destruction (25). Moreover, a progression of radiological damages occurs between the acute and chronic phase of PIA, like in RA where radiographic progression can follow a linear or a sigmoid curve (25).

In RA, ED was observed both in macro- and in microcirculation (26) but to date, the course of these two impairments is still debated. In this study, endothelial function was explored by measuring the vasorelaxant response of isolated vessels to acetylcholine, an endothelium- 
dependent vasorelaxant agonist. The data showed impaired response to Ach reflecting ED both in macro- (aorta) and microcirculation (mesenteric arteries) in PIA, the course of ED being vascular bed-dependent. Whereas ED in macrocirculation was present in acute phase but reversible in chronic phase, the opposite observation was made for microcirculation. These results are congruent with a few studies in RA that compared micro/macrovascular endothelial function in the same patient, at the same time, and that did not find any correlation (26,27). From a diagnosis perspective, these data indicate that the presence of ED in microcirculation cannot be used for predicting ED in large arteries at a given time. This dissociation in the courses of ED among vascular beds suggests that pathophysiological mechanisms are distinct, that deserve to be elucidated. In mesenteric arteries harvested in the chronic phase of PIA, Ach induced a biphasic response showing relaxation at the lower concentrations and contractions at the higher concentrations (Figure 3F). This vascular response is identical to that observed in vessels from spontaneously hypertensive rats (28), and in aortic rings from rats with mono-arthritis (29) in which it has been attributed to the endothelial production of cyclo-oxygenase (COX)-derived contracting factors and superoxide anion generation. Thus, it would be interesting to explore whether the same mechanisms are involved in PIA model to determine if the production of endotheliumderived constricting factors is a common feature of the arthritis-associated vascular dysfunction. An interesting result of our study is that vascular dysfunction was not restricted to the endothelium but also targeted the sensitivity of VSMC to vasoconstrictive mediators. Indeed, our results showed the presence of a decreased vasoconstrictive response, mainly to adrenergic agonists, restricted to macrovascular bed, predominant in acute phase, and resulting from both abnormalities of VSMC reactivity and ED. To note, such impairments have already been observed in the early stage of the mice CIA model with 5-HT (10) and in the rat AIA model with norepinephrine (30). The clinical relevance of such dysfunction 
remains to be understood, as well as whether such dysfunction is specific to animal models or could also occur in RA patients.

The link between clinical inflammation (assessed by disease activity scores) and biological inflammation (assessed by blood levels of C-reactive protein, pro-inflammatory cytokines or erythrocyte sedimentation rate) and $\mathrm{ED}$ in RA, albeit highly suspected, has not been demonstrated, and conflicting results have been obtained. From our study in PIA, it appears that one hypothesis for this controversy might be that macro- and microcirculation respond differently to inflammation. Indeed, we demonstrated that macrovascular ED correlated with disease activity and systemic inflammation whilst microvascular ED did not. This result is consistent with previously observed associations between the improvement in macrovascular endothelial function and the reduction in disease activity after treatment with biologic agents $(31,32)$. Moreover, the lack of association between disease activity and microvascular ED was already described (33). Again, these data suggest that mechanisms involved in ED at both vascular levels are distinct. In support of this are the results showing that treatment with abatacept transiently improved microvascular endothelial function while progressively deteriorating macrovascular endothelial function (34). To date, no valuable biomarker of ED has been identified in RA $(35,36)$. A few studies suggested that circulating lipids $(37)$, adiponectin (38) or MPO levels (37) correlated with endothelial function. In our study, none of these markers correlated with endothelial function whatever the vascular bed except levels of total cholesterol that were positively correlated with macrovascular endothelial function. As cholesterol levels are dependent on the inflammatory burden in RA (7), this result is consistent with the positive correlation observed between macrovascular endothelial function and systemic inflammation and suggest a deleterious effect of inflammation on macrovascular endothelial function. Our data revealed that the PIA model is associated with a sustained altered lipid profile, indicating that this model mimics the "lipid paradox" in RA (7). Only a 
few studies investigated lipid levels in animal models of arthritis. Whereas the AIA model did not exhibit lipid impairments (13), low cholesterol levels were measured in rat (39) and mice CIA (40) models. However, compared to these "acute" monophasic models, the longstanding alteration in lipid profile from the acute to the chronic phase makes of PIA a relevant model for long-term studies on lipid disorders in RA.

In conclusion, the novel finding provided by this study is that the PIA model reproduces several features of the $\mathrm{CV}$ alterations occurring in RA: ED in micro- and macrocirculation with independency of course and mechanisms among these vascular beds, a link between inflammation and macrovascular ED, and low lipid levels. These data suggest that this model would be very useful for long-term pharmacological studies as well for deciphering the complex pathophysiology of increased CV risk in RA. Further studies are needed to determine whether this model might also mimic the cardiac dysfunction associated with RA.

Disclosure of interest: The authors declare that they have no competing of interest.

Financial support: This work was supported by funding from the French Ministry for Higher Education and Research.

Acknowledgements: The authors thank Mrs M. Tournier-Nappey for the IL-6, adiponectin, total cholesterol and triglycerides levels measurements.

Appendix A. Supplementary data

Supplementary data (Figures S1-S3) associated with this article can be found in the online version at ... 


\section{References}

1. Prati C, Demougeot C, Guillot X, Sondag M, Verhoeven F, Wendling D. Vascular involvement in axial spondyloarthropathies. Joint Bone Spine 2018. doi: 10.1016/j.jbspin.2018.05.003

2. Prasad M, Hermann J, Gabriel SE, Weyand CM, Mulvagh S, Mankad R, et al. Cardiorheumatology: cardiac involvement in systemic rheumatic disease. Nat Rev Cardiol. 2015;12(3):168-76.

3. Avina-Zubieta JA, Thomas J, Sadatsafavi M, Lehman AJ, Lacaille D. Risk of incident cardiovascular events in patients with rheumatoid arthritis: a meta-analysis of observational studies. Ann Rheum Dis. 2012;71(9):1524-9.

4. del Rincón ID, Williams K, Stern MP, Freeman GL, Escalante A. High incidence of cardiovascular events in a rheumatoid arthritis cohort not explained by traditional cardiac risk factors. Arthritis Rheum. 2001;44(12):2737-45.

5. Bordy R, Totoson P, Prati C, Marie C, Wendling D, Demougeot C. Microvascular endothelial dysfunction in rheumatoid arthritis. Nat Rev Rheumatol. 2018;14(7):404-20.

6. Moroni L, Selmi C, Angelini C, Meroni PL. Evaluation of Endothelial Function by Flow-Mediated Dilation: a Comprehensive Review in Rheumatic Disease. Arch Immunol Ther Exp (Warsz). 2017; 65:463-75.

7. Choy E, Sattar N. Interpreting lipid levels in the context of high-grade inflammatory states with a focus on rheumatoid arthritis: a challenge to conventional cardiovascular risk actions. Ann Rheum Dis. 2009;68(4):460-9. 
8. van den Hoek J, Boshuizen HC, Roorda LD, Tijhuis GJ, Nurmohamed MT, van den Bos $\mathrm{G}$ a. M, et al. Mortality in patients with rheumatoid arthritis: a 15-year prospective cohort study. Rheumatol Int. 2017;37(4):487-93.

9. Boissier M-C, Bessis N. Do we need animal models to advance research on inflammatory joint disease? Jt Bone Spine Rev Rhum. 2017;84(4):381-3.

10. Reynolds S 1, Williams AS, Williams H, Smale S, Stephenson HJ, Amos N, et al. Contractile, but not endothelial, dysfunction in early inflammatory arthritis: a possible role for matrix metalloproteinase-9. Br J Pharmacol. 2012;167(3):505-14.

11. Palma Zochio Tozzato G, Taipeiro EF, Spadella MA, Marabini Filho P, de Assis MR, Carlos CP, et al. Collagen-induced arthritis increases inducible nitric oxide synthase not only in aorta but also in the cardiac and renal microcirculation of mice. Clin Exp Immunol. 2016;183(3):341-9.

12. Totoson P, Maguin-Gaté K, Prati C, Wendling D, Demougeot C. Mechanisms of endothelial dysfunction in rheumatoid arthritis: lessons from animal studies. Arthritis Res Ther. 2014;16(1):202.

13. Totoson P, Maguin-Gaté K, Prigent-Tessier A, Monnier A, Verhoeven F, Marie C, et al. Etanercept improves endothelial function via pleiotropic effects in rat adjuvant-induced arthritis. Rheumatol Oxf Engl. 2016;55(7):1308-17.

14. Tuncel J, Haag S, Hoffmann MH, Yau ACY, Hultqvist M, Olofsson P, et al. Animal Models of Rheumatoid Arthritis (I): Pristane-Induced Arthritis in the Rat. PloS One. 2016;11(5):e0155936. 
15. Hoffmann MH, Tuncel J, Skriner K, Tohidast-Akrad M, Türk B, Pinol-Roma S, et al. The rheumatoid arthritis-associated autoantigen hnRNP-A2 (RA33) is a major stimulator of autoimmunity in rats with pristane-induced arthritis. J Immunol Baltim Md 1950. 2007;179(11):7568-76.

16. Wernhoff $\mathrm{P}$, Olofsson $\mathrm{P}$, Holmdahl R. The genetic control of rheumatoid factor production in a rat model of rheumatoid arthritis. Arthritis Rheum. 2003;48(12):3584-96.

17. Leichsenring A, Bäcker I, Furtmüller PG, Obinger C, Lange F, Flemmig J. Long-Term Effects of (-)-Epigallocatechin Gallate (EGCG) on Pristane-Induced Arthritis (PIA) in Female Dark Agouti Rats. PloS One. 2016;11(3):e0152518.

18. Hou W, Meng L, Zheng F, Wen Y, Zhu W, Jiang C, et al. Methotrexate ameliorates pristane-induced arthritis by decreasing IFN- $\gamma$ and IL-17A expressions. J Zhejiang Univ Sci B. 2011;12(1):40-6.

19. Ackerman NR, Rooks WH, Shott L, Genant H, Maloney P, West E. Effects of naproxen on connective tissue changes in the adjuvant arthritic rat. Arthritis Rheum. 1979;22(12):1365-74.

20. Bradley PP, Priebat DA, Christensen RD, Rothstein G. Measurement of cutaneous inflammation: estimation of neutrophil content with an enzyme marker. J Invest Dermatol. 1982;78(3):206-9.

21. Vingsbo C, Sahlstrand P, Brun JG, Jonsson R, Saxne T, Holmdahl R. Pristane-induced arthritis in rats: a new model for rheumatoid arthritis with a chronic disease course 
influenced by both major histocompatibility complex and non-major histocompatibility complex genes. Am J Pathol. 1996;149(5):1675-83.

22. Peters MJL, Symmons DPM, McCarey D, Dijkmans B a. C, Nicola P, Kvien TK, et al. EULAR evidence-based recommendations for cardiovascular risk management in patients with rheumatoid arthritis and other forms of inflammatory arthritis. Ann Rheum Dis. 2010;69(2):325-31.

23. Totoson P, Maguin-Gaté K, Nappey M, Prati C, Wendling D, Demougeot C. Microvascular Abnormalities in Adjuvant-Induced Arthritis: Relationship to Macrovascular Endothelial Function and Markers of Endothelial Activation. Arthritis Rheumatol. 2015;67(5):1203-13.

24. Le Goff B, Soltner E, Charrier C, Maugars Y, Rédini F, Heymann D, et al. A combination of methotrexate and zoledronic acid prevents bone erosions and systemic bone mass loss in collagen induced arthritis. Arthritis Res Ther. 2009;11(6):R185.

25. Morel J, Combe B. How to predict prognosis in early rheumatoid arthritis. Best Pract Res Clin Rheumatol. 2005;19(1):137-46.

26. Sandoo A, Carroll D, Metsios GS, Kitas GD, Veldhuijzen van Zanten JJ. The association between microvascular and macrovascular endothelial function in patients with rheumatoid arthritis: a cross-sectional study. Arthritis Res Ther. 2011;13(3):R99.

27. Sandoo A, Chanchlani N, Hodson J, Smith JP, Douglas KM, Kitas GD. Classical cardiovascular disease risk factors associate with vascular function and morphology in rheumatoid arthritis: a six-year prospective study. Arthritis Res Ther. 2013;15(6):R203. 
28. Félétou M, Verbeuren TJ, Vanhoutte PM. Endothelium-dependent contractions in SHR: a tale of prostanoid TP and IP receptors. Br J Pharmacol. 2009;156(4):563-74.

29. Hamilton K, Dunning L, Ferrell WR, Lockhart JC, MacKenzie A. Endothelium-derived contraction in a model of rheumatoid arthritis is mediated via angiotensin II type 1 receptors. Vascul Pharmacol. 2018;100:51-7.

30. Totoson P, Maguin-Gaté K, Nappey M, Wendling D, Demougeot C. Endothelial Dysfunction in Rheumatoid Arthritis: Mechanistic Insights and Correlation with Circulating Markers of Systemic Inflammation. PloS One. 2016;11(1):e0146744.

31. Hürlimann D, Forster A, Noll G, Enseleit F, Chenevard R, Distler O, et al. Anti-tumor necrosis factor-alpha treatment improves endothelial function in patients with rheumatoid arthritis. Circulation. 2002;106(17):2184-7.

32. Gonzalez-Juanatey C, Llorca J, Sanchez-Andrade A, Garcia-Porrua C, Martin J, Gonzalez-Gay MA. Short-term adalimumab therapy improves endo-thelial function in patients with rheumatoid arthritis refractory to infliximab. Clin Exp Rheumatol. 2006;24(3):309-12.

33. Hänsel S, Lässig G, Pistrosch F, Passauer J. Endothelial dysfunction in young patients with long-term rheumatoid arthritis and low disease activity. Atherosclerosis. $2003 ; 170(1): 177-80$.

34. Sandoo A, Kitas GD. The impact of abatacept treatment on the vasculature in patients with rheumatoid arthritis. Clin Exp Rheumatol. 2015;33(4):589. 
35. Di Minno MND, Ambrosino P, Lupoli R, Di Minno A, Tasso M, Peluso R, et al. Clinical assessment of endothelial function in patients with rheumatoid arthritis: A meta-analysis of literature studies. Eur J Intern Med. 2015;26(10):835-42.

36. Klimek E, Skalska A, Kwaśny-Krochin B, Surdacki A, Sulicka J, Korkosz M, et al. Differential associations of inflammatory and endothelial biomarkers with disease activity in rheumatoid arthritis of short duration. Mediators Inflamm. 2014;2014:681635.

37. Mäki-Petäjä KM, Cheriyan J, Booth AD, Hall FC, Brown J, Wallace SML, et al. Inducible nitric oxide synthase activity is increased in patients with rheumatoid arthritis and contributes to endothelial dysfunction. Int J Cardiol. 2008;129(3):399-405.

38. Komai N, Morita Y, Sakuta T, Kuwabara A, Kashihara N. Anti-tumor necrosis factor therapy increases serum adiponectin levels with the improvement of endothelial dysfunction in patients with rheumatoid arthritis. Mod Rheumatol. 2007;17(5):385-90.

39. Srivastava NK, Sharma S, Purusottam RN, Sinha N, Singh R, Sharma D. Abnormal lipid metabolism in collagen-induced arthritis rat model: in vitro, high resolution NMR spectroscopy based analysis. Indian J Exp Biol. 2014;52(7):673-82.

40. Wen W, He M, Liang X, Gao S, Zhou J, Yuan Z. Accelerated transformation of macrophage-derived foam cells in the presence of collagen-induced arthritis mice serum is associated with dyslipidemia. Autoimmunity. 2016;49(2):115-23. 


\section{Figure Legends}

\section{Figure 1. Clinical and radiological features in the PIA model.}

Evolution of arthritis score (A) and body weight (B) in PIA compared to control rats. Radiographic score in acute phase (C) and chronic phase (D). PIA-A: PIA group in acute phase; CONT-A: control group in acute phase; PIA-C: PIA group in chronic phase; CONT-C: control group in chronic phase; $* * \mathrm{p}<0.001$.

Figure 2. Reactivity to vasoconstrictors in macro- and microvascular bed in the PIA model.

Endothelium-intact aortic rings were constricted with cumulative concentrations of Phe in acute (A) and chronic (B) phases, and of 5-HT in acute (C) and chronic (D) phases of the PIA model. The same experiments were conducted in mesenteric arteries vasoconstricted with Phe in acute (E) and chronic (F) phases, and with 5-HT in acute $(\mathrm{G})$ and chronic $(\mathrm{H})$ phases of PIA. PIA-A: PIA group in acute phase; CONT-A: control group in acute phase; PIA-C: PIA group in chronic phase; CONT-C: control group in chronic phase; n: number of vessels; NS: non-significant; $* \mathrm{p}<0.05 ; * * \mathrm{p}<0.001 ; * * * \mathrm{p}<0.0001$.

Figure 3. Reactivity to endothelium-dependent and -independent vasodilators in macroand microvascular beds in the PIA model.

Endothelium-intact aortic rings pre-constricted with 5-HT were dilated with cumulative concentrations of acetylcholine (Ach) in acute (A) and chronic (B) phases, or with the NO donor sodium nitroprussiate (SNP) in acute (C) and chronic (D) phases of the PIA model. Likewise, endothelium-intact mesenteric arteries pre-constricted with Phe were dilated with 
cumulative concentrations of Ach in acute (E) and chronic (F) phases, or with SNP in acute (G) and chronic $(\mathrm{H})$ phases of the PIA model. PIA-A: PIA group in acute phase; CONT-A: control group in acute phase; PIA-C: PIA group in chronic phase; CONT-C: control group in chronic phase; n: number of vessels; NS: non-significant; * $\mathrm{p}<0.05$; ** $\mathrm{p}<0.001$. 
A

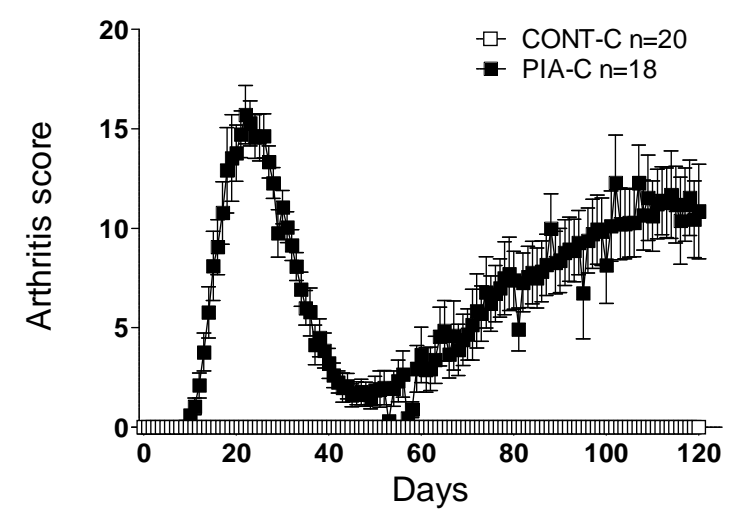

C

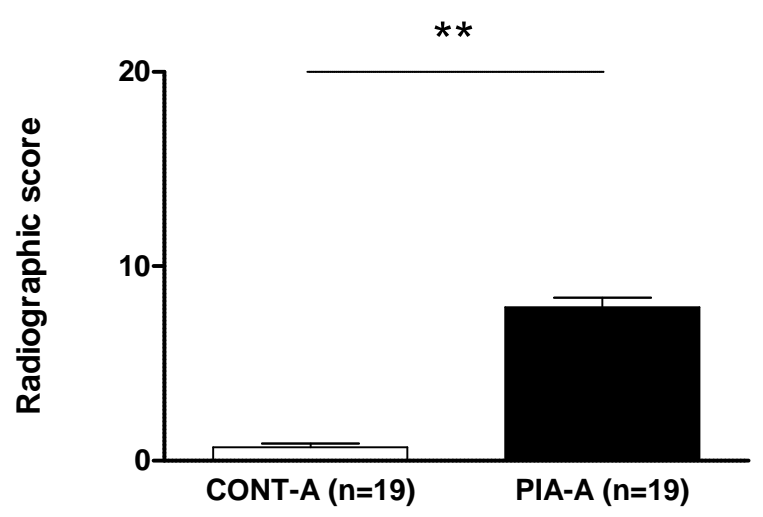

B

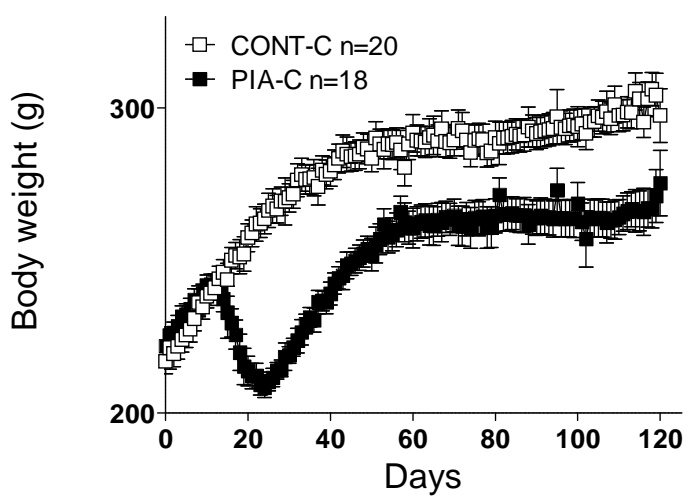

$\mathrm{D}$

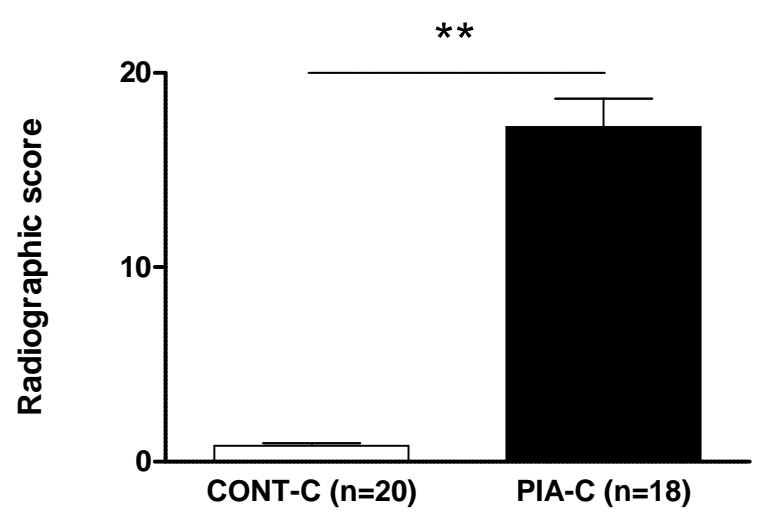

Figure 1. Clinical and radiological features in the PIA model. 
A

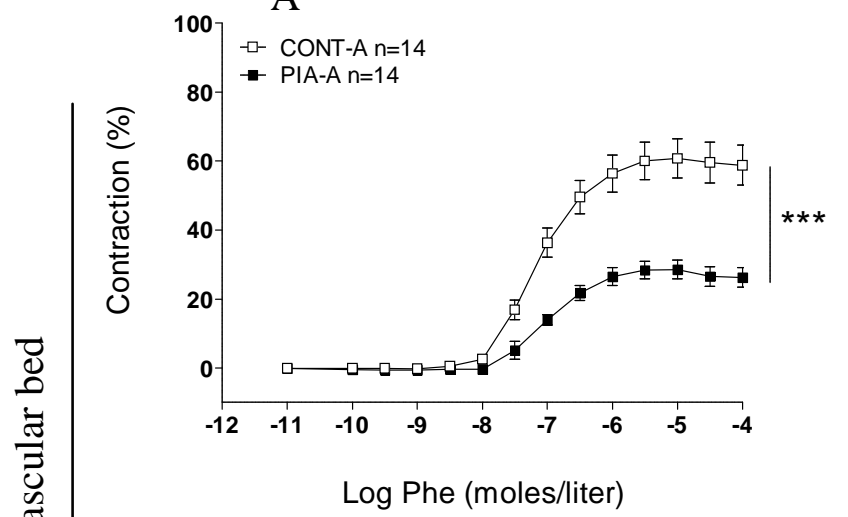

C

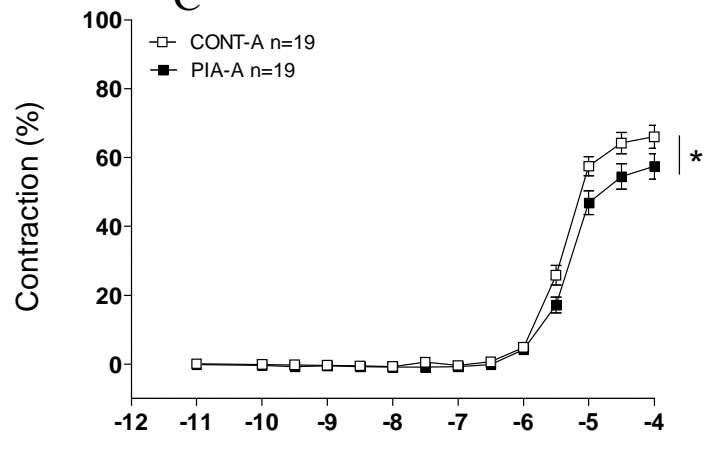

Log 5-HT (moles/liter)

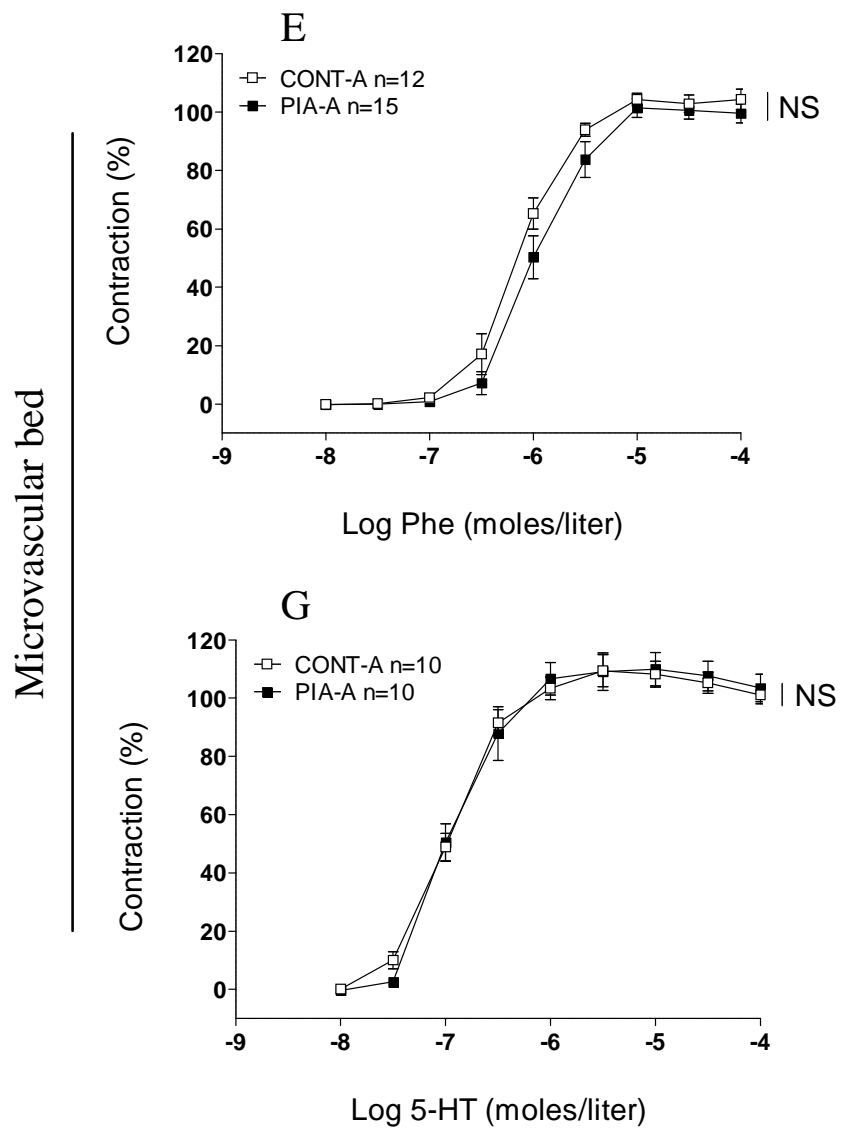

Chronic phase

B

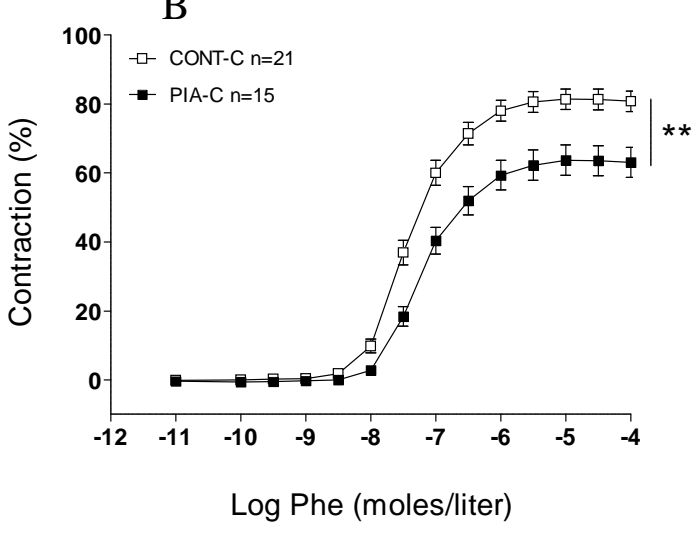

D

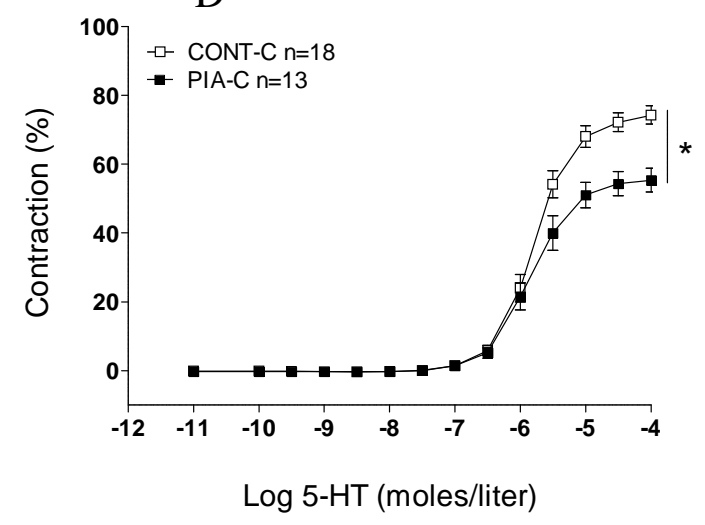

F

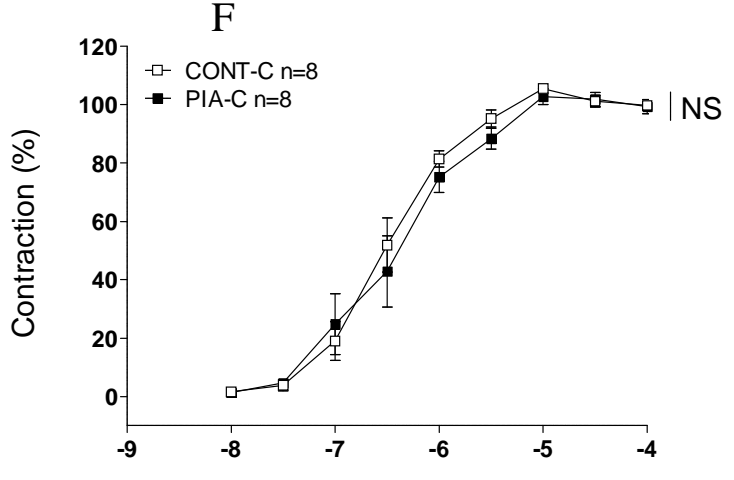

Log Phe (moles/liter)

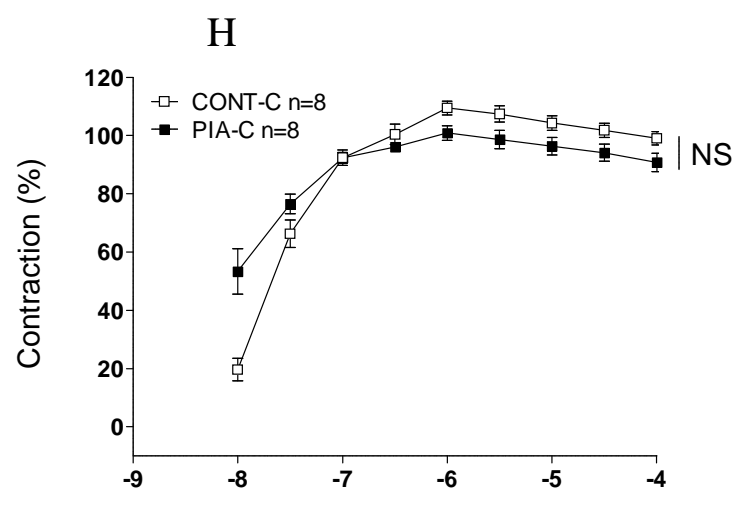

Log 5-HT (moles/liter) 
Figure 2. Reactivity to vasoconstrictors in macro- and microvascular bed in the PIA model. 
Acute phase

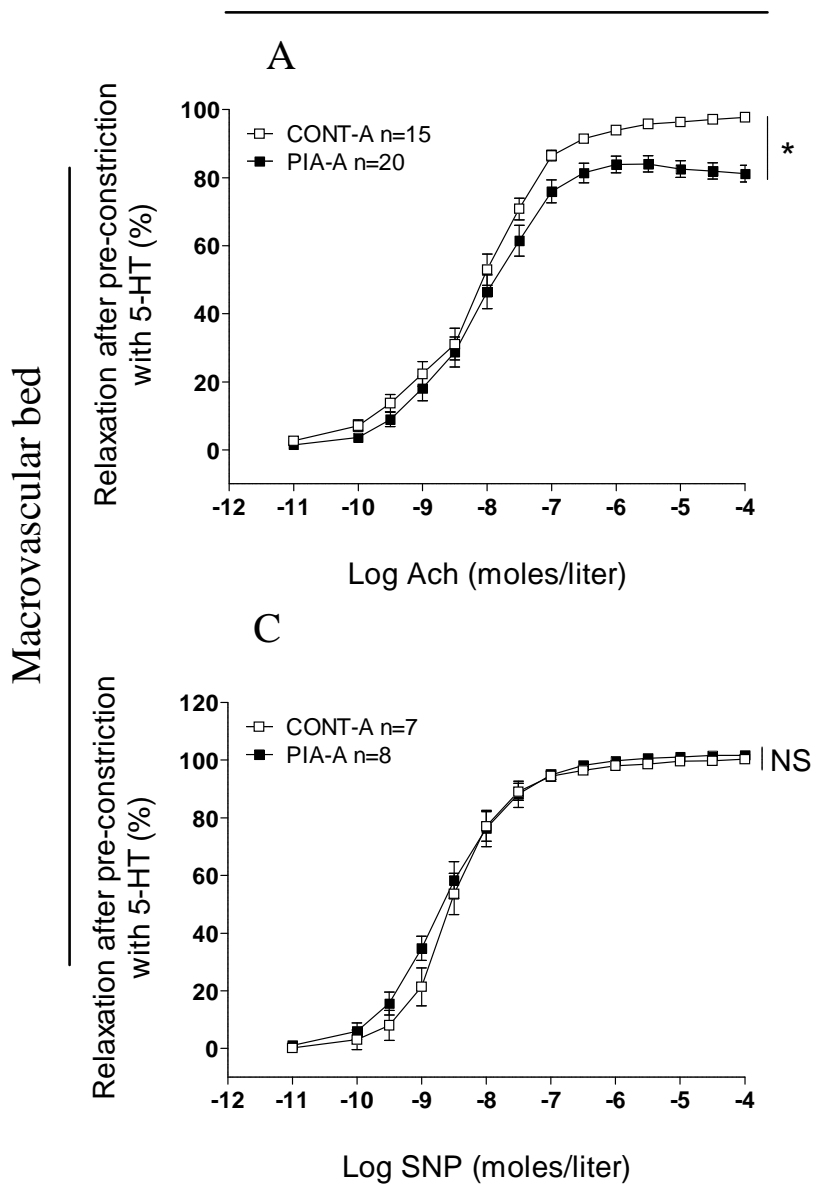

E

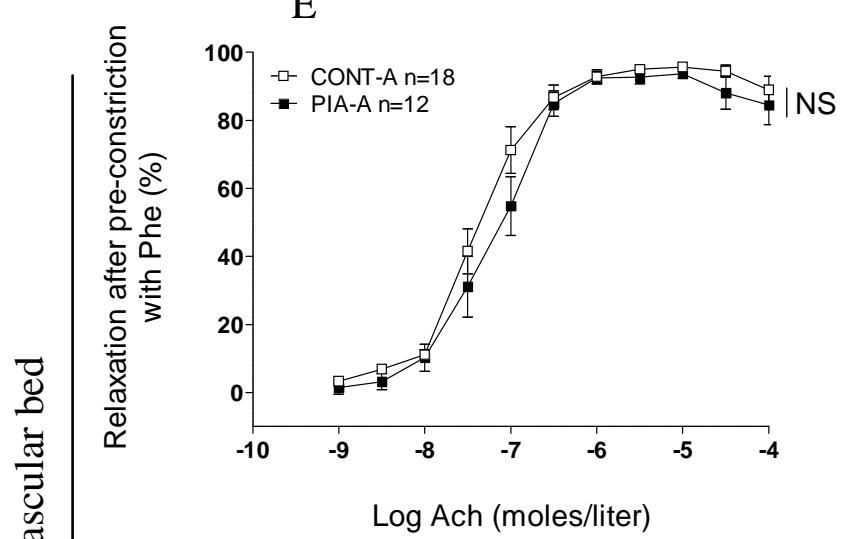

G

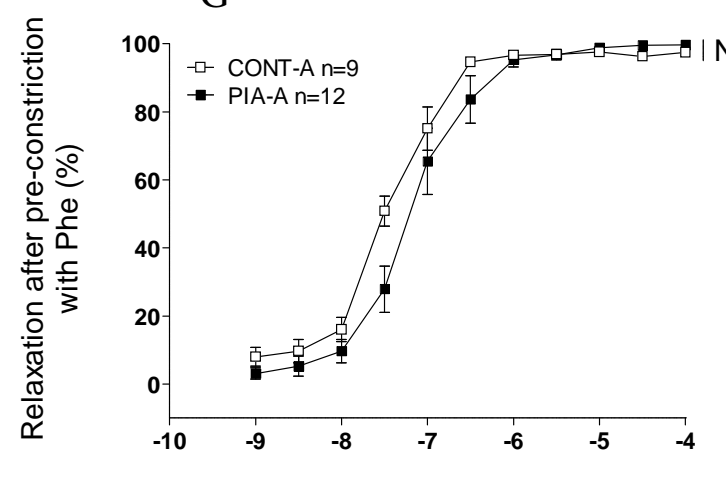

Log SNP (moles/liter)
Chronic phase

B

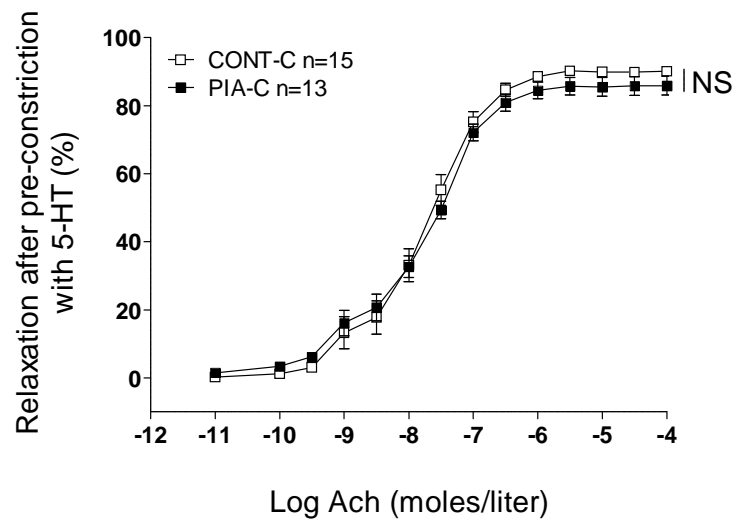

D

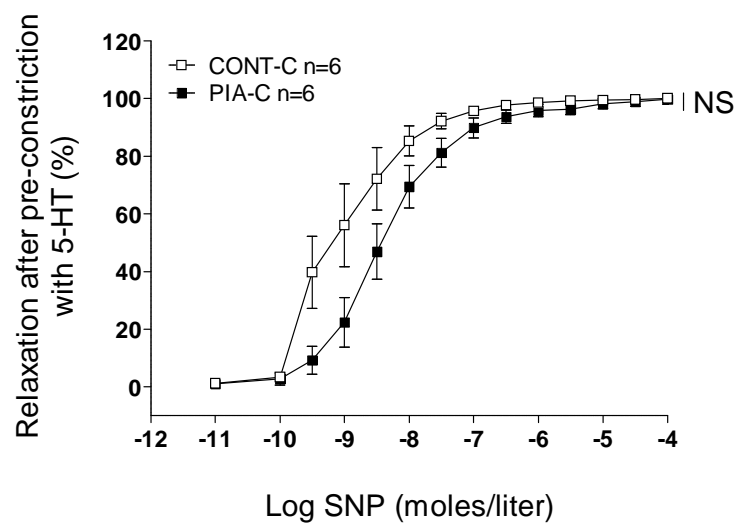

F

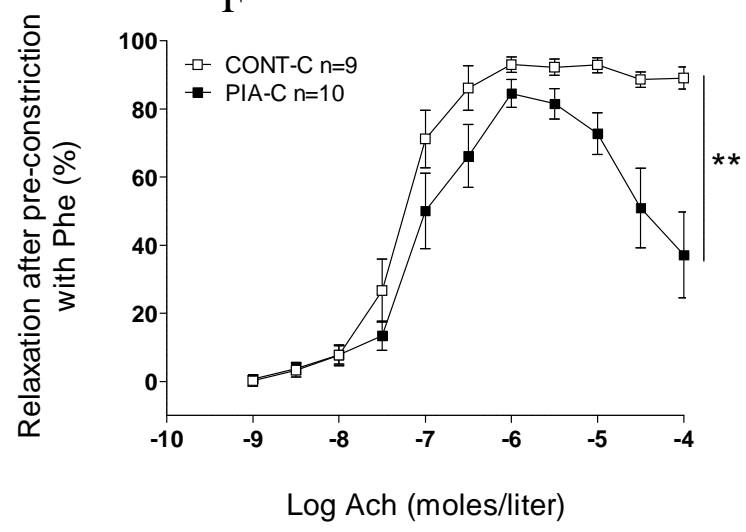

$\mathrm{H}$

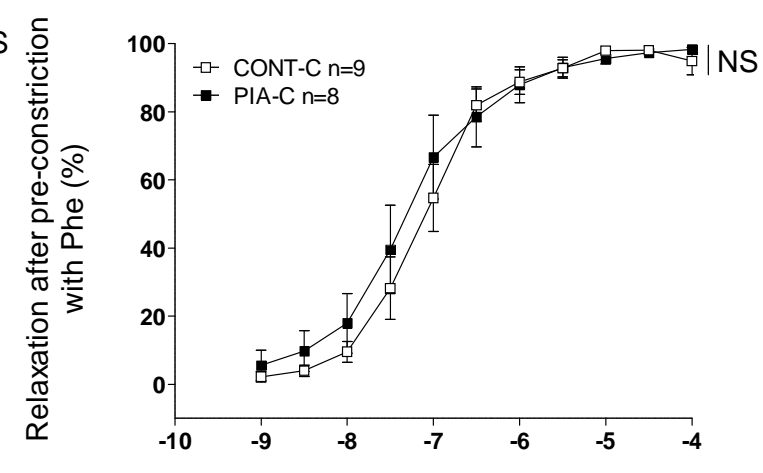

Log SNP (moles/liter) 
Figure 3. Reactivity to endothelium-dependent and -independent vasodilators in macroand microvascular beds in the PIA model. 
Table 1. Description of arthritis phases in the PIA model.

\begin{tabular}{lcccc}
\hline & \multicolumn{2}{c}{ Acute phase } & \multicolumn{2}{c}{ Chronic phase } \\
\hline & PIA-A & PIA-C & PIA-A & PIA-C \\
\hline$\%^{\text {a }}$ & 100 & 95 & n.a. & 77.8 \\
Onset (day) $^{\mathrm{b}}$ & $12.4 \pm 1.9$ & $13.3 \pm 3.3$ & n.a. & $73.8 \pm 19.2$ \\
Maximal arthritis score (day) $^{\mathrm{c}}$ & $18.7 \pm 2.7$ & $21.4 \pm 2.5$ & n.a. & 120 \\
Maximal arthritis score (score) $^{\mathrm{d}}$ & $20.1 \pm 5.0$ & $17.3 \pm 4.6$ & n.a. & $15.5 \pm 6.5$ \\
Weight loss (\%) $^{\mathrm{e}}$ & $17.5 \pm 3.1$ & $16.7 \pm 3.5$ & n.a. & n.a. \\
Radiographic score (score) & $7.9 \pm 2.2$ & n.a. & n.a. & $17.2 \pm 6.1$ \\
\hline
\end{tabular}

"a" percentage of rats presenting an arthritis phase; "b" Mean day of onset; "c" Mean day of maximal arthritis score; "d" Mean maximal arthritis score; "e" Mean maximum weight loss compared to maximal weight at onset; n.a.: not applicable; PIA-A: PIA group in acute phase; PIA-C: PIA group in chronic phase. Values are means \pm SEM ( $\mathrm{N}=14$ to 20 rats per group). 
Table 2. Effects of PIA on circulating markers.

\begin{tabular}{|c|c|c|c|c|}
\hline & \multicolumn{2}{|c|}{ Acute phase } & \multicolumn{2}{|c|}{ Chronic phase } \\
\hline & Control & PIA & Control & PIA \\
\hline IL-6 (pg/ml) & $107.1 \pm 26.4$ & $210.0 \pm 63.5 * *$ & $114.5 \pm 73.2$ & $243.0 \pm 76.0 * *$ \\
\hline $\begin{array}{l}\text { Myeloperoxidase activity } \\
\qquad(\mathrm{UI} / \mathrm{ml})\end{array}$ & $3.2 \pm 1.3$ & $3.9 \pm 1.6$ & $3.0 \pm 0.9$ & $3.7 \pm 1.3$ \\
\hline Blood glucose $(\mathrm{g} / \mathrm{l})$ & $1.1 \pm 0.1$ & $1.1 \pm 0.1$ & $1.0 \pm 0.1$ & $1.0 \pm 0.1$ \\
\hline Triglycerides $(\mathrm{mmol} / \mathrm{ml})$ & $1.4 \pm 0.3$ & $1.0 \pm 0.2 * *$ & $1.4 \pm 0.4$ & $0.8 \pm 0.2 * *$ \\
\hline Total cholesterol $(\mathrm{mg} / \mathrm{ml})$ & $1.6 \pm 0.1$ & $1.4 \pm 0.1 * *$ & $1.5 \pm 0.1$ & $1.3 \pm 0.1 * *$ \\
\hline Adiponectin $(\mu \mathrm{g} / \mathrm{ml})$ & $8.1 \pm 2.0$ & $4.7 \pm 1.6 * *$ & $8.3 \pm 1.8$ & $8.0 \pm 1.8$ \\
\hline
\end{tabular}

Values are means $\pm \mathrm{SEM}(\mathrm{N}=14$ to 20 rats per group). $* *(p<0.001)$ versus corresponding control. 